\title{
Quantum mechanical aspect of first order phase transition of crystals
}

\author{
J. Kobayashi ${ }^{\mathrm{a}}$ \\ Department of Applied Physics, Waseda University, Shinjuku-ku, Tokyo, 169-8555, Japan
}

Received 12 March 2010 / Received in final form 27 May 2010

Published online 16 July 2010 - (C) EDP Sciences, Società Italiana di Fisica, Springer-Verlag 2010

\begin{abstract}
It has been generally believed that some external physical porperties, e.g. volume, enthalpy, and entropy, etc. change discontinuously at first order phase transition temperatures, since Ehrenfest's proposition. However, the deviation from this proposition was often found in many crystals. As the progress of experimental methods and the accuracy develop the number of crystals that manifest unusual transition processes is increasing. Notably aberrant phenomena are as follows. An intermediate phase appears whose crystal structure is undoubtedly different from those of the low and high temperature forms. The peak of differential thermal analysis of specific heat is splitted into two as if one transition inevitably induces another. The interpretation of these abnormal behaviors in the vicinity of the transition is certainly beyond reach of thermodynamic ideas. We assumed that the eigenkets of Boltzmann's $H$ of each phase in the vicinity of the transition temperature interact to produce perturbing state. Then the intermediate phase named $\mathrm{M}$ phase emerges, and its eigenket is the superposition of eigenkets of commuting Hamiltonian of the two temperature phases. It is natural that the new $M$ phase has different structure from those of the two phases. The above mentioned phenomena occurring in dichlorobenzophenone, $\mathrm{NaNO}_{2}, 1$-Ethyl-3-(4methylpentanoyl)urea, and $\mathrm{VO}_{2}$ are explained by this quantum mechanical theory.
\end{abstract}

\section{Introduction}

Gibbs free energy $G$ of low (1) and high (2) temperature forms of a crystal, that is functions of temperature $T$ and pressure $P$ is expressed by curved planes in a regular $T$ and $P$ space. The intersection of these two planes draws a single curved line indicating common $G$ values. In a special case of a constant $P$, the intersection line breaks into two different lines crossing at certain temperature $T_{o}$, where $G$ of the two phases coincide. In this case, a transformation can takes place from one phase to another reversibly. The order of such a phase transformation in solids was defined by Ehrenfest [1]; it is the same as the derivative of the Gibbs free energy which show a discontinuous change at the transition. Volume, enthalpy, and entropy etc. are the typical thermodynamical quantities exhibiting first order transition, and thermal expasivity and heat capacity are those of the second order transition.

The general features of a first order phase transition, which is abbreviated 'FOPT' in what follows, are known as follows. (i) In the equilibrium state of the two phases at $T_{o}$ the transition occurs reversibly from the smaller entropy phase to the higher entropy one. As the change of entropy is discontinuous, the latent heat develops. (ii) The change of the fraction of the two phases is discontinuous. (iii) The transitions exhibit generally hysteresis

\footnotetext{
a e-mail: jkob@kd6.so-net.ne.jp
}

phenomenon. These phenomena can be perfectly understood by thermodynamical theories.

It is important to note that these general conditions are not always exactly held in the real FOPTs as reviewed comprehensively by Herbstein [2]. Discrepancies of real phenomena against thermodynamical theories were already noticed in days of 1950's by pioneer workers. Especially Ubbelohde [3] found an intermediate phase appearing in the process of FOPT between the two phases. Mnyukh [4] put forward a theory that the FOPTs are completed by nucleation-to-growth mechanism. Therefore the crystal is always inhomogeneous in the process of FOPTs. It is worth-stressing that in accordance with the progress of experimental methods and accuracy abnormal facts have been more revealed in FOPTs. Especially it needs emphasis that most of aberrant phenomena in FOPT seem entirely unintelligible by thermodynamical view points. We will survey difficult problems of FOPT in the next chapter, and try to elucidate origins of these effects on quantum mechanical ground in the later chapters.

\section{Problems of first order phase transition in crystals}

Accurate knowledges of FOPT in crystals cannot be obtained without having overcome various experimental difficulties. Especially care must be paid to keep constant 


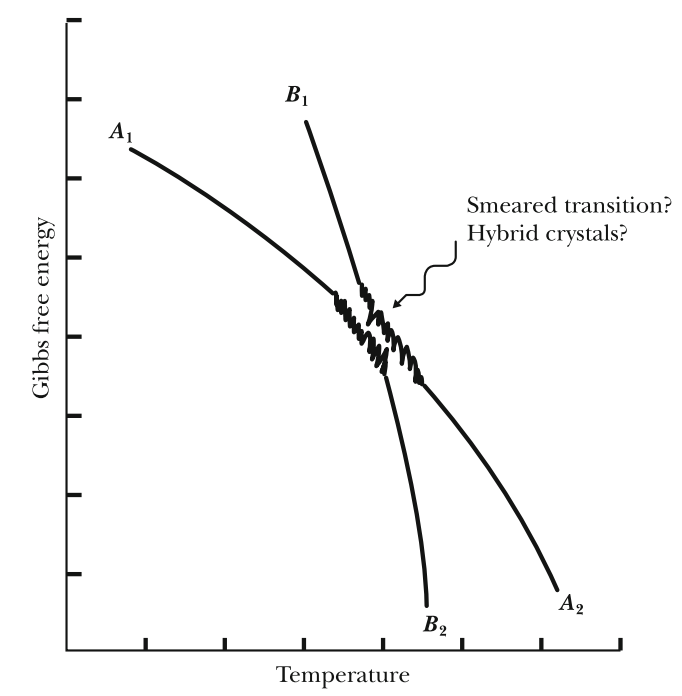

Fig. 1. Smeared free energy in the vicinity of transition point of low and high temperature phases (after Herbsten [2]).

temperatures accurately for long time, and to avoid local coagulation of constituent components in the body. However, it has been confirmed that the following phenomena are often observed even under sufficiently careful treatments.

(i) Generation of an intermediate phase which is appreciably different from 1 and 2 phases in the process of FOPT. Ubbelohde [3] drew attention on the premonitory phenomena preceding FOPT and found a specific intermediate phase to form between 1 and 2 phases, on which he named 'hybrid crystal' or 'smeared transition'. The hybrid crystal is consisted of sub-units associated with cells of 1 and 2 phases fulfilling the characteristics of a single crystal on average. As the temperature moves away from the transformation point, the distinguishable sub-units disappear. In essence the hybrid crystal seems independent from 1 and 2 phases but resembled to be a mixing medium of them. Ubbelohde struggled to interpret the formation of hybrid crystal on the thermodynamical stand, and reached a concept that $G_{1}$ and $G_{2}$ lines become smeared near $T_{o}$ as shown in Figure 1. Rao and Rao [5] suggested that the aforementioned free energy planes of both phases might swell when $T_{o}$ is approached. At any rate, this effect cannot be given rational solutions from macroscopic ideas.

(ii) Nucleation and growth mechanism. Mnyukh [4] investigated the process of observations that the transformation proceeds mostly as inhomogeneous media. Mnyukh's idea certainly explains the characteristic property of FOPTs, viz. transformation hysteresis accompanied by superheating and supercooling. On the other hand, Gooding and Morris [6] assured theoretically that in the case of FOPT, the fluctuation of density of the transforming phase does not increase when the transition temperature is approached.

It is natural to consider that the nucleation of a new phase in the body of the host phase requires ad hoc

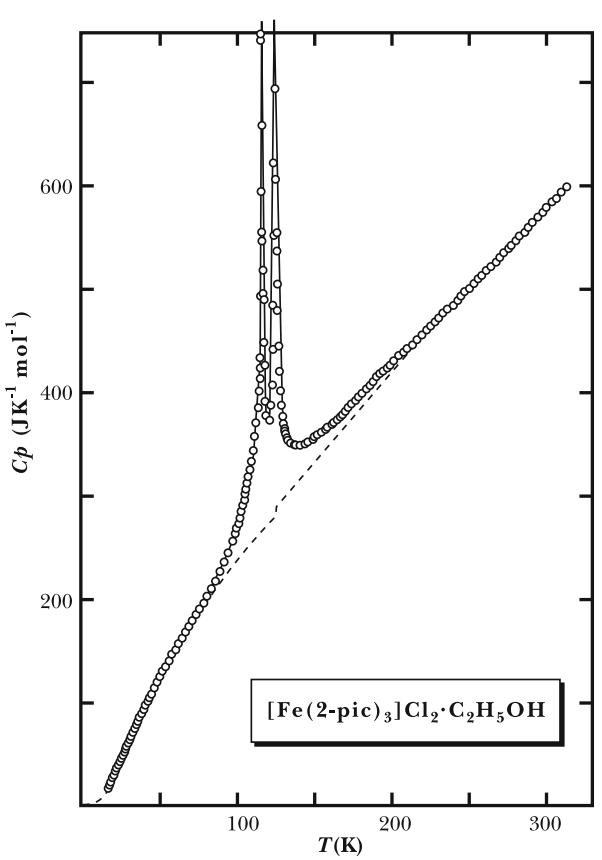

Fig. 2. Temperature dependence of specific heat of $[\mathrm{Fe}(2-$ pic) $\left.{ }_{3}\right] \mathrm{Cl}_{2}-\mathrm{C}_{2} \mathrm{H}_{5} \mathrm{OH}$ (after Herbstein [2]).

strain energy $s$ and internal surface energy $w$. Let the common free energy of 1 and 2 phases in process of FOPT is defined as

and

$$
G_{\mid}=G\left(P, T, s_{12}, w_{12}\right) \quad \text { for superheating, }
$$

$$
G_{\|}=G\left(P, T, s_{21}, w_{21}\right) \quad \text { for supercooling. }
$$

It is readily understood that owing to the presence of additive energy $s$ and $w$, transitions take place different temperatures from original $T_{o}$. Thus it appears that Mnyukh's theory assists to understand one characteristic nature of FOPTs, but is unable to sustain the criticism on the aforementioned inhomogeneity of crystal media.

(iii) Aberration of physical properties on FOPT. Aberrant physical properties are found in certain crystals that are unintelligible by the thermodynamical idea. (a) Physical properties of the intermediate phases are unambiguously not the mixture of those of the constituent 1 and 2 phases. (b) A peak of specific heat around $T_{o}$ is splitted into two with different extents of separation. One example of $\mathrm{Fe}(2 \text {-pic })_{3} \mathrm{Cl}_{2}$-EtOH is shown in Figure 2. Then a serious conclusion is likely to be led that a FOPT is apt to induce another one. (c) It appears appreciably that the crystal structure of the interleaved phase is different from those of any one of 1 and 2 phases.

The reasonable explanations of these unusual phenomena have not yet been given. It seems to us that there is scarcely possibility to resolve these phenomena on the thermodynamical basis. It appears worth-while to consider them on quantum mechanical view point, since the phase transitions of crystal are essentially related to change of microscopic atomic motions. 
3 Quantum mechanical analysis of first order phase transition

\subsection{Basic theory}

A dynamical state $\phi(q)$ of the systems in a quantum mechanical ensemble containing the similar non-interacting systems is generally represented by a linear combination of the eigenkets of any other observable, where $q$ is coordinate observable. The density operator $\rho$ of an ensemble reads,

$$
\rho=\sum_{i}\left|\Psi^{i}\right\rangle p_{i}^{2}\left\langle\Psi^{i}\right|
$$

$\left|\Psi^{i}\right\rangle$ is an eigenket of $\rho(i=1,2, \ldots, n, \ldots)$, and $p_{i}^{2}$ is the eigenvalue belonging to it. Physically $P_{i}=p_{i}^{2}$ indicates the weight of finding $\left|\Psi^{i}\right\rangle$ in the ensemble. In accordance with above statement, $\left|\Psi^{i}\right\rangle$ can be expressed by the linear combination of eigenkets, $\left|u_{m}\right\rangle,\left|u_{n}\right\rangle$..., etc. belonging to any arbitrary observable $\xi$ of the ensemble. That is to say,

$$
\left|\Psi^{i}\right\rangle=\sum_{n} c_{n}^{i}\left|u_{n}\right\rangle
$$

where $c_{n}^{i}=\left\langle\Psi^{i} \mid u_{n}\right\rangle$ is the probability amplitude of $n$ state of $\xi$. Let take the representation of $\rho$ by using the basic kets of $\left.|n\rangle \equiv u_{n}\right\rangle \ldots$ etc. One of a diagonal component $\rho_{n n}$ of the density matrix is written

$$
\rho_{n n}=\left\langle n\left|\sum_{c}\right| \Psi^{i}\right\rangle P_{i}\left\langle\Psi^{i} \mid n\right\rangle=\sum_{i} P_{i} c_{n}^{i} c_{n}^{i *}
$$

which is an eigenvalue of $\xi$ expressed by using eigenvalues of density matrix.

Now define $a_{n}$ as

$$
a_{n}=\sum_{i}\left\langle n\left|p_{i}\right| \Psi^{i}\right\rangle=\sum_{i} p_{i} c_{n}^{i} .
$$

Then $W_{n}=\left(a_{n} a_{n}^{*}\right)$ means the probability of finding a member of the ensemble in the $n$ eigenstate of $\xi$. If $N$ systems are contained in the ensemble, a components of density matrix is ensemble averaged value. For instance,

$$
\rho_{n n}=\frac{1}{N} \sum_{\alpha=1}^{N} a_{n}^{\alpha} a_{n}^{\alpha *}=\overline{\overline{a_{n} a_{n}^{*}}}
$$

We use a single bar to denote a mean value which has been obtained by the single process of averaging over the range of probabilities corresponding to quantum mechanical state of a single system, and to use a double bar to denote a mean value which has been obtained by the process of averaging over the systems of an ensemble. Let diagonal component of $\rho$ matrix be defined as $R$, for example,

$$
R_{n}=\rho_{n n}=\overline{\bar{W}}_{n}=\overline{\overline{a_{n} a_{n}^{*}}} .
$$

Then the following relation holds in an ensemble.

$$
\xi|n\rangle=R_{n}|n\rangle
$$

As a useful function of the density operator, it produces ensemble average value of any observable. Taking $A$ observable as an example,

$$
\overline{\bar{A}}=\sum_{m n} A_{m n} \rho_{n m} .
$$

This relation, matrix multiplication of the two matrices, is rewritten in the form

$$
\overline{\bar{A}}=\operatorname{Tr} A P=\operatorname{Tr} P A .
$$

In order to see analogs between quantum mechanical quantities and corresponding thermodynamic ones, we derive first the canonical density matrix. The condition, under which the systems will remain in equilibrium, i.e. appear unaltered under the elapse of time, requires from Heisenberg equation,

$$
[\mathcal{H}, \rho]=0
$$

where $\mathcal{H}$ is the Hamiltonian operator of the system. In another word $\rho$ must be a function of $\mathcal{H}$. The proof is given: we argue that the two separate systems are combined into composite system, whose Hamiltonian is just the sum of the separate Hamiltonians. On the other hand, the density operator of the composite system is a product of the separate density operators of the two systems. It follows that the relationship between $\rho$ and $\mathcal{H}$ must be of the functional form

$$
\rho=e^{X / \theta} e^{-\mathcal{H} / \theta},
$$

where $X$ and $\theta$ are parameters. Thus the density operator is a function of Hamiltonian. Then canonical density ensemble fulfils a satisfactory condition of being in a equilibrium state. It is natural that

$$
\sum_{n} R_{n}=\sum_{n} e^{\frac{X-E_{n}}{\theta}}=1
$$

where $E_{n}$ is an internal energy of the $n$ complexion of the Hamiltonian of the ensemble. The mean internal energy of an ensemble is written by using (10).

$$
\overline{\bar{E}}=\sum_{n} R_{n} E_{n}=\sum_{n} e^{\frac{X-E_{n}}{\theta}} E_{n}
$$

We consider now the ensemble average of Boltzmann's $H$ of both 1 and 2 phases. Definition of $\overline{\bar{H}}$ is given by

$$
\overline{\bar{H}}=\sum_{n} R_{n} \ln R_{n}
$$

Namely, $\overline{\bar{H}}$ is an ensemble-average of $\ln R . \overline{\bar{H}}$ of an ensemble can measure deviation from equilibrium and tends to decrease with time, reaching finally a minimum value. By (13) and (15), an important relation is obtained

$$
\overline{\bar{H}}=\sum_{n} e^{\frac{X-E_{n}}{\theta}} \frac{X-E_{n}}{\theta}=\frac{X-\overline{\bar{E}}}{\theta} .
$$


Furthermore, from (13)

$$
e^{-X / \theta}=\sum_{n} e^{-E_{n} / \theta}=Z
$$

and

$$
X / \theta=-\ln \sum_{n} e^{-E_{n} / \theta}=-\ln Z
$$

where $Z$ is the partition function. On the other hand, from (16)

$$
\delta \overline{\bar{H}}=\frac{\delta X}{\theta}-\frac{\delta \overline{\bar{E}}}{\theta}-\frac{X-\overline{\bar{E}}}{\theta^{2}} \delta \theta .
$$

From (13)

$$
\delta \sum_{n} R_{n}=\delta \sum_{n} e^{\frac{X-E_{n}}{\theta}}=0 .
$$

If not only $X$ and $\theta$, but also relevant external coordinate $q_{i}$ is assumed to change slightly, $\overline{\bar{E}}$ will be consequently altered, i.e. (20) becomes

$$
\begin{aligned}
\sum_{n} e^{\frac{X-E_{n}}{\theta}\left\{\frac{\delta X}{\theta}-\frac{1}{\theta}\left(\frac{\partial E_{n}}{\partial q_{1}} \delta q_{1}\right.\right.} & \left.+\frac{\partial E_{n}}{\partial q_{2}} \delta q_{2}+\ldots\right) \\
& \left.-\frac{X-E_{n}}{\theta^{2}} \delta \theta\right\}=0 .
\end{aligned}
$$

For any state $n$, the generalized external forces $A_{i}$ are defined as

$$
A_{1}=-\frac{\partial E_{n}}{\partial q_{1}}, \quad A_{2}=-\frac{\partial E_{n}}{\partial q_{2}}, \ldots
$$

Naturally

$$
\overline{\overline{A_{1}}}=\sum_{n} e^{\frac{X-E_{n}}{\theta}} A_{1}, \quad \overline{\overline{A_{2}}}=\sum_{n} e^{\frac{X-E_{n}}{\theta}} A_{2}, \ldots
$$

Then, (19) becomes

$$
\frac{\delta X}{\theta}+\frac{1}{\theta}\left(\overline{\overline{A_{1}}} \delta q_{1}+\overline{\overline{A_{2}}} \delta q_{2}+\ldots\right)-\frac{X-\overline{\bar{E}}}{\theta^{2}} \delta \theta=0 .
$$

From (19) and (21)

$$
\begin{aligned}
-\delta \overline{\bar{H}} & =\frac{\overline{\bar{E}}}{\theta}-\left(\frac{\delta X}{\theta}-\frac{-\delta \overline{\bar{E}}}{\theta^{2}} \delta \theta\right) \\
& =\frac{\delta \overline{\bar{E}}}{\theta}+\frac{1}{\theta}\left(\overline{\overline{A_{1}}} \delta q_{1}+\overline{\overline{A_{2}}} \delta q_{2}+\ldots\right) .
\end{aligned}
$$

In a case that the external forces are not applied to the system, (25) becomes

$$
-\delta \overline{\bar{H}}=\frac{\delta \overline{\bar{E}}}{\theta} .
$$

On the other hand, thermodynamic theory states that the change of entropy $S$ is expressed as

$$
\delta S=\frac{\delta E}{T}+\frac{1}{T}\left(A_{1} \delta q_{1}+A_{2} \delta q_{2}+\ldots\right),
$$

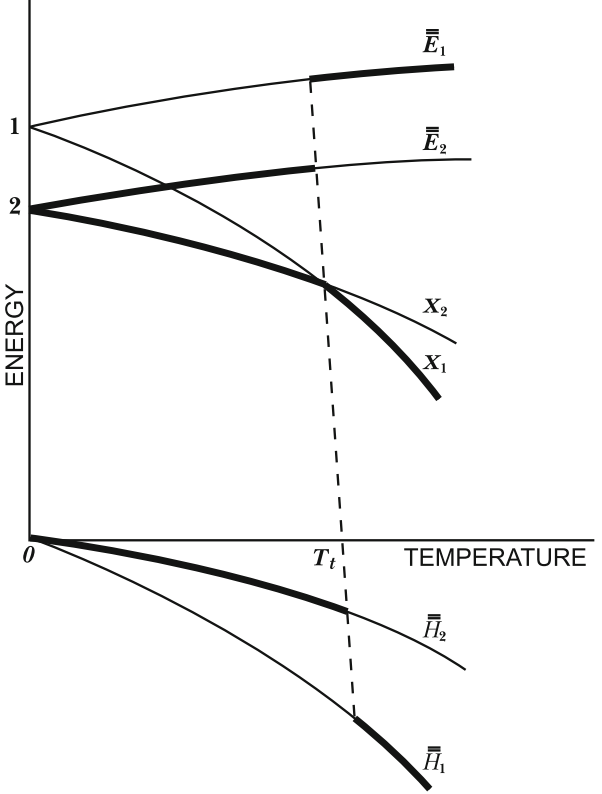

Fig. 3. Thermal variation of internal energy $\overline{\bar{E}}$, Helmholtz's free energy $X$, and Boltzmann's $\overline{\bar{H}}$ function of crystals.

where $E$ is the internal energy. The similarity between (25) and (27) indicates correlations of entropy $S$ and temperature $T$ with statistical mechanical quantities $-\overline{\bar{H}}$ and $\theta$, viz.

$$
S \Leftrightarrow \kappa \overline{\bar{H}}, \quad \text { and } \quad T \Leftrightarrow \theta / \kappa,
$$

where the mark $\Leftrightarrow$ means 'correlate'. A constant $\kappa$ with the dimension of energy over temperature has to be introduced to allow for difference in units. From (15)

$$
X=\overline{\bar{E}}+\theta \overline{\bar{H}}
$$

On the other hand, a thermodynamical relation reads

$$
A=E-T S
$$

where $A$ is the Helmholtz free energy. Then we can mark the correlation

$$
A \Leftrightarrow X
$$

In accordance with (18), $X$ is related with partition function as

$$
X=-\kappa T \ln Z=-\kappa T \ln \sum_{n} e^{-\frac{E_{n}}{\kappa T}}
$$

\subsection{Perturbation theory of first order phase transition}

We regard the dynamical state of our crystal to be separated into two quantum mechanical ensembles corresponding to 1 and 2 phases. In Figure 3 temperature dependence of mean internal energy of both ensembles $\overline{\overline{E_{1}}}$, and $\overline{\overline{E_{2}}}$ are drawn. Assume that $\overline{\overline{E_{1}}}>\overline{\overline{E_{2}}}$ at $T=0$ and both increase 
gradually with increase of temperature. $X$ of both ensembles coincide with each internal energy at $T=0$, and decrease with increase of $T, X_{2}<X_{1}$ in the low temperature region, but both curves intersect at $T_{t}$ and $X_{2}>X_{1}$ afterward. $\overline{\overline{H_{1}}}$ and $\overline{\overline{H_{2}}}$ take in each ensemble minimum value under each $\overline{\overline{E_{1}}}$ and $\overline{\overline{E_{2}}}$ energy. Their temperature variations are shown in Figure 3 . It is important to note that the two partition functions $Z_{1}$ and $Z_{2}$ become equal at $T_{t}$, where the 1 ensemble becomes a special state by joining the 2 ensemble. Total $\overline{\overline{\mathcal{H}}}$ of this ensemble changes reversibly into $\overline{\overline{H_{1}}}$ under the partition function. Let the final state of 1 ensemble be named as ' $\mathrm{M}$ ' ensemble or phase.

The original eigenkets of $\overline{\overline{H_{1}}}$ are designated as $\phi_{1}, \ldots$ $\phi_{n}, \ldots$ etc, which are taken as a basis for representation of the density matrix of $\mathrm{M}$ ensemble. With regard to an arbitrary eigenstate $n$, say, of $\overline{\overline{H_{1}}}$, the Schrödinger equation holds as can be inferred from (8).

$$
\overline{\overline{H_{1}}} \phi_{n}=R_{n} \phi_{n}
$$

The story so far holds under the condition that eigenstates of $\overline{\overline{H_{1}}}$ and $\overline{\overline{H_{2}}}$ are perfectly orthonormal. Now we feel it probable and so assume that there exists interaction between them which produces eventually a perturbing energy $V$ for the final state $\overline{\overline{H_{1}}}$ or M phase. This idea may relate to the macroscopic phenomenon of free energy planes of both phases becoming thicker as the temperature approaches $T_{t}$ as has already been stated. Then (33) becomes

$$
\overline{\overline{H_{1}^{\prime}}} \varphi_{n}=R_{n}^{\prime} \varphi_{n}
$$

where

$$
\overline{\overline{H_{1}^{\prime}}}=\overline{\overline{H_{1}}}+V / \kappa
$$

and

$$
R_{n}^{\prime}=R_{n}+R_{n}^{(1)} .
$$

Here it is noted that the eigenfunctions of $\overline{\bar{H}}$ and $\overline{\overline{\mathcal{H}}}$ operators form a complete set. Thus the eigenkets $\phi_{n}^{(i)}(i=1,2)$ of $\overline{\overline{\mathcal{H}}}$ are degenerated with $\phi_{n}$ with a common eigenvalue of $R_{n}$. Then $\varphi_{n}$ is written as

$$
\varphi_{n}=\sum_{i} \alpha_{i} \phi_{n}^{(i)}+\sum_{b \neq n} C_{n b} \sum_{i} \beta_{i} \phi_{b}^{(i)}
$$

here $\phi_{n}^{(i)}$ are orthonormal eigenfunctions. $\alpha_{i}$ and $\beta_{i}$ are relative weights among $\phi_{n}^{(i)}$ and $\phi_{b}^{(i)}$, and $C_{n b}$ is the interaction coefficient between $\varphi_{n}$ and $\phi_{b}^{(i)}$. By using (34) and (36), the equation in the perturbed state reads

$$
\begin{gathered}
\left(\overline{\overline{H_{1}}}+V / \kappa\right)\left\{\sum_{i} \alpha_{i} \phi_{n}^{(i)}+\sum_{b \neq n} C_{n b} \sum_{i} \beta_{i} \phi_{b}^{(i)}\right\}= \\
\left(R_{n}+R_{n}^{(1)}\right)\left\{\sum_{i} \alpha_{i} \phi_{n}^{(i)}+\sum_{b \neq n} C_{n b} \sum_{i} \beta_{i} \phi_{b}^{(i)}\right\} .
\end{gathered}
$$

The equation of first order in smallness in (37) is written

$$
\begin{aligned}
\overline{\overline{H_{1}}} \sum_{b \neq n} C_{n b} \sum_{i} \beta_{i} \phi_{b}^{(i)}+V / \kappa \sum_{i} \alpha_{i} \phi_{n}^{(i)}= \\
R_{n} \sum_{b \neq n} C_{n b} \sum_{i} \beta_{i} \phi_{b}^{(i)}+R_{n}^{(1)} \sum_{i} \alpha_{i} \phi_{n}^{(i)} .
\end{aligned}
$$

In order to express this equation by using Heisenberg representation, take the scaler product of $\phi_{b}^{(j)}$ with (38). The first order shift equation becomes

$$
\begin{aligned}
& \overline{\overline{H_{1}}} \phi_{n}^{(j)} \sum_{b \neq n} C_{n b} \sum_{i} \beta_{i} \phi_{b}^{(i)}+\phi_{n}^{(j)} V / \kappa \sum_{i} \alpha_{i} \phi_{n}^{(i)}= \\
& \phi_{n}^{(j)} R_{n} \sum_{b \neq n} C_{n b} \sum_{i} \beta_{i} \phi_{b}^{(i)}+\phi_{n}^{(j)} R_{n}^{(1)} \sum_{i} \alpha_{i} \phi_{n}^{(i)} .
\end{aligned}
$$

By the condition that $\left\langle\phi_{n}^{(i)} \mid \phi_{b}^{(j)}\right\rangle=\delta_{n b} \delta_{i j}$ (39) becomes

$$
\sum_{i} \alpha_{i}\left\langle\phi_{n}^{(j)}|V / \kappa| \phi_{b}^{(i)}\right\rangle=\alpha_{j} R_{n}^{(1)} .
$$

This is a two-dimensional eigenvalue equation. As there is a two-fold degeneracy, a simplified abbreviation is used.

$$
\left\langle\phi_{n}^{(j)}|V / \kappa| \phi_{n}^{(i)}\right\rangle=h_{j i}
$$

Then (40) can be written

$$
\begin{aligned}
& \alpha_{1} h_{11}+\alpha_{2} h_{12}=\alpha_{1} R_{n}^{(1)} \\
& \alpha_{1} h_{21}+\alpha_{2} h_{22}=\alpha_{2} R_{n}^{(1)} .
\end{aligned}
$$

For real $V, h_{j i}$ can be represented by an Hermitian matrix with eigenvalues $R_{n i}^{(1)}$, which can be obtained by solving the determinant,

$$
\left|\begin{array}{cc}
h_{11}-R_{n}^{(1)} & h_{12} \\
h_{21} & h_{21}-R_{n}^{(1)}
\end{array}\right|=0 .
$$

Two eigenvalues are obtained,

and

$$
\begin{aligned}
& R_{n 1}^{(1)}=\frac{1}{2}\left(h_{11}+h_{22}\right)-\frac{1}{2}\left\{\left(h_{11}-h_{22}\right)+4 h_{12}^{2}\right\}^{1 / 2} \\
& R_{n 2}^{(1)}=\frac{1}{2}\left(h_{11}+h_{22}\right)+\frac{1}{2}\left\{\left(h_{11}-h_{22}\right)+4 h_{12}^{2}\right\}^{1 / 2}
\end{aligned}
$$

(42) can be expressed in terms of two matrix equations for the two eigenvalues $R_{n i}$ and vector matrices $\left(\alpha_{1 i}, \alpha_{2 i}\right)$.

$$
\left(\begin{array}{ll}
h_{11} & h_{12} \\
h_{12} & h_{22}
\end{array}\right)\left(\begin{array}{l}
\alpha_{11} \\
\alpha_{21}
\end{array}\right)=R_{n 1}^{(1)}\left(\begin{array}{l}
\alpha_{11} \\
\alpha_{21}
\end{array}\right),
$$

and

$$
\left(\begin{array}{ll}
h_{11} & h_{12} \\
h_{12} & h_{22}
\end{array}\right)\left(\begin{array}{l}
\alpha_{12} \\
\alpha_{22}
\end{array}\right)=R_{n 2}^{(1)}\left(\begin{array}{l}
\alpha_{12} \\
\alpha_{22}
\end{array}\right) .
$$

In other words, $h_{j i}$ matrices have the principal vectors $\mathbf{r}_{1}$ $\left(\alpha_{11}, \alpha_{21}\right)$ and $\mathbf{r}_{2}\left(\alpha_{12}, \alpha_{22}\right)$, belonging to eigenvalues of $R_{n 1}^{(1)}$ and $R_{n 2}^{(1)}$ respectively. A general figure representing 


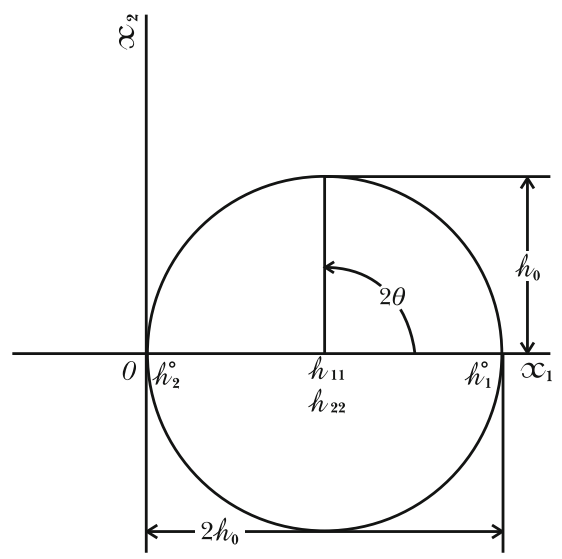

(a)

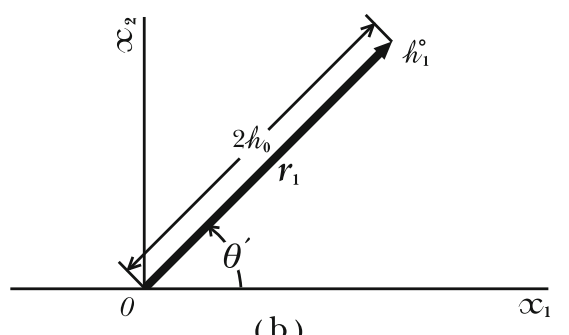

(b)

Fig. 4. Mohr circle construction of the representation quadric of perturbing energy matrix at $T_{t}$.

$h_{i j} x_{i} x_{j}=1$ in the rectangular coordinate system $\left(x_{1}, x_{2}\right)$ is a quadric, i.e. an ellipse in the present case. The principal vectors $\mathbf{r}_{1}$ and $\mathbf{r}_{2}$ of an ellipse are added to produce a superposed vector $\mathbf{r}=\alpha_{1} \mathbf{r}_{1}+\alpha_{2} \mathbf{r}_{2}$ which correspond to the dynamical state of $\mathrm{M}$ ensemble, direction of $\mathbf{r}$ is, of course, different from those of $\mathbf{r}_{1}$, and $\mathbf{r}_{2}$, and the length is determined by distribution coefficients $\alpha_{1}$ and $\alpha_{2}$.

\subsection{Thermal change of distribution coefficients around $T_{t}$}

It would be of primary importance to evaluate the distribution coefficients in the structure of the $\mathrm{M}$ phase at $T_{t} \cdot \overline{\bar{H}}_{1}$ and $\overline{\overline{\mathcal{H}}}$ operators have degerate eigenfunctions $\phi_{n}$ and $\phi_{n}^{(i)}$ at $T_{t}$. However, it can be considered that the two eigenfunctions $\phi_{n}^{(1)}$ and $\phi_{n}^{(2)}$ of $\overline{\overline{\mathcal{H}}}$ are entirely indistinguishable in the $\mathrm{M}$ phase since two equal partition functions exist there. As the result, it would be correct to regard that $h_{11}, h_{22}$ and $h_{12}$ are represented by the same component $h_{0}$ say. The lengths and inclination angles $\theta$ of $\mathbf{r}_{1}$ and $\theta+90^{\circ}$ of $\mathbf{r}_{2}$ of the principal axes can be obtained by using the Mohr circle construction as shown in Figure $4 \mathrm{a}$, where $R_{n 1}^{(1)}=2 h_{0}$ and $R_{n 2}^{(1)}=0$ from (44). The lengths of the principal axes defined by $h_{1}^{0}$ and $h_{2}^{0}$ are marked at $2 h_{0}$ and zero positions in $x_{1}$ axis. The principal axes of the ellipse derived from this construction are shown in Figure 4b. They become a straight line with half length of $2 h_{0}$ and $\theta=45^{\circ}$. Therefore the total inclination

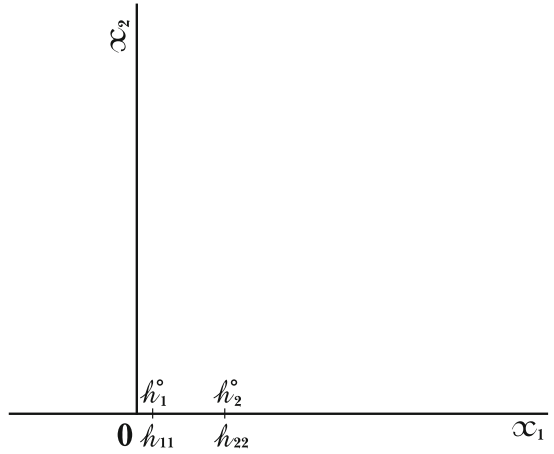

(a)

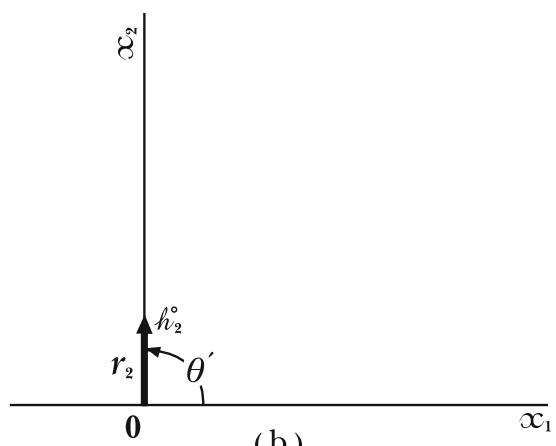

( b )

Fig. 5. Mohr circle construction of the representation quadric of perturbing energy matrix slightly above $T_{l}$.

angle $\theta^{\prime}$ of the vector $\mathbf{r}$, i.e. $\theta^{\prime}=\theta+\delta$ is $45^{\circ}$, where $\delta$ is the angle formed by the principal axes of the ellipse, viz. $\delta=\tan ^{-1} h_{2}^{0} / h_{1}^{0}=0$. Thus $\alpha_{1}=\cos 45^{\circ}=1 / \sqrt{2}$ and $\alpha_{1}=\sin 45^{\circ}=1 / \sqrt{2}$.

In view of the physical continuity, it would be correct to infer that the weak perturbing energy $V$ begins to appear from slightly lower temperature $T_{l}$ than $T_{t}$, and afterward it fades away in slightly higher temperature $T_{h}$, the width of the total interval $\Delta T$ of the $\mathrm{M}$ phase including $T_{t}$ being usually very small.

Around $T_{l}, \phi_{n}^{(2)}$ will be dominant over $\phi_{n}^{(1)}$ which is almost negligible. We can take that $h_{11}=h_{1}^{0} \sim 0, h_{22}=$ $h_{2}^{0}, h_{12} \sim 0$, and $\theta \sim 0$. The Mohr circle construction is shown in Figure 5a in enlarged scale. The resultant ellipse is indicated in Figure 5b, where $\theta=0$ and $\delta=\tan ^{-1} \infty=$ $90^{\circ}$. Therefore $\theta^{\prime}=\delta=90^{\circ}$. Thus $\alpha_{1}=\cos \theta^{\prime} \sim 0$, and $\alpha_{2}=\sin \theta^{\prime}=1$.

In the temperature interval between $T_{l}$ and $T_{t}, V$ increases appreciably, so $h_{11}$ becomes discernible, while $h_{22}$ decreases. Therefore the distance between $h_{11}$ and $h_{22}$ is shortened as shown in Figure 6a for the Mohr circle construction, where the principal axes $h_{1}^{0}, h_{2}^{0}$, and $2 \theta$ are marked. The resultant ellipse is indicated in Figure $6 \mathrm{~b}$ by the principal axes. $\theta^{\prime}$ is larger than $45^{\circ}$. Thus $\alpha_{1}=\cos \theta^{\prime}=\cos (\theta+\delta)$, and $\alpha_{2}=\sin \theta^{\prime}=\sin (\theta+\delta)$.

In the temperature range between $T_{t}$ and $T_{h}$, the similar construction is indicated in Figure 7a, where the relative positions of $h_{11}$ and $h_{22}$ are reversed. $\theta^{\prime}=\theta+\delta$ becomes smaller than $45^{\circ}$ as found in Figure $7 \mathrm{~b}$. Thus $\alpha_{1}=\cos \theta^{\prime}=\cos (\theta+\delta)$ and $\alpha_{2}=\sin \theta \prime=\sin (\theta+\delta)$. 


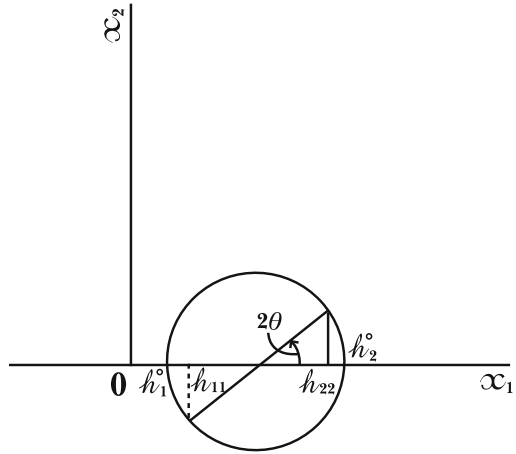

(a)

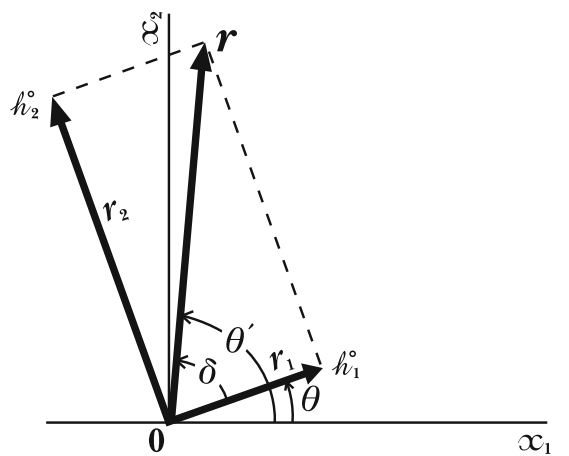

(b)

Fig. 6. Mohr circle construction of the representation quadric of perturbing energy matrix between $T_{l}$ and $T_{t}$.

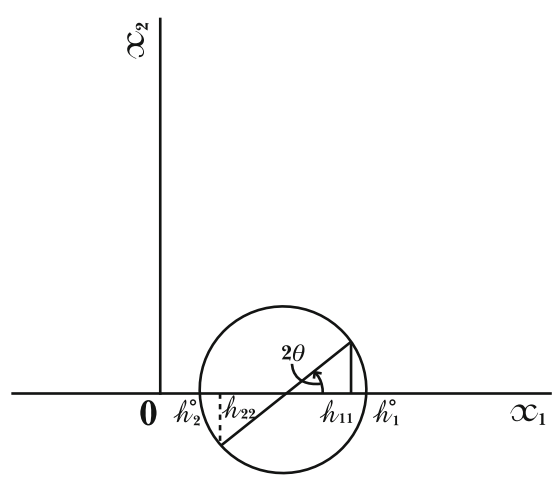

(a)

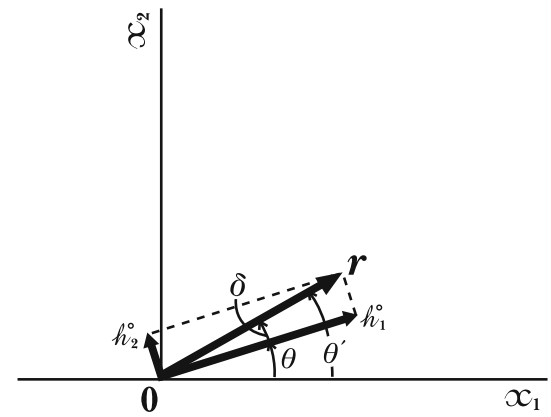

( b )

Fig. 7. Mohr circle construction of the representation quadric of perturbing energy matrix between $T_{t}$ and $T_{h}$.

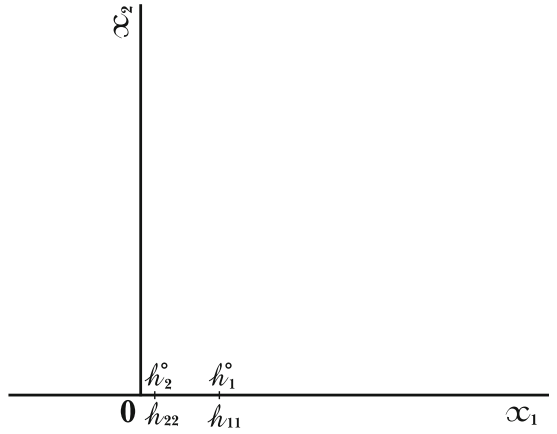

(a)

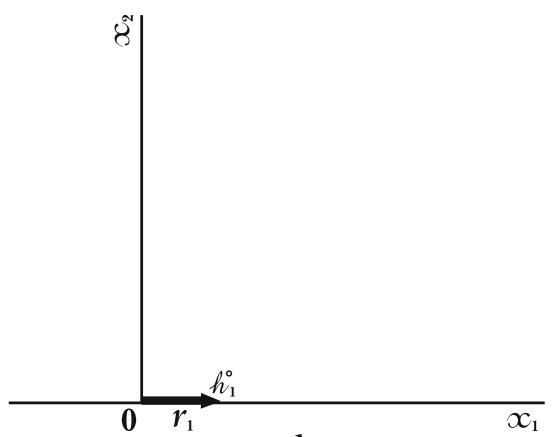

( b )

Fig. 8. Mohr circle construction of the representation quadric of perturbing energy matrix slightly below $T_{h}$.

At temperature immediately below $T_{h}$, the similar construction is indicated in Figure 8a, where $h_{11}=h_{1}^{0}, h_{22}=$ $h_{2}^{0} \sim 0$, and $\theta=0$. The ellipse becomes a straight line as shown in Figure 8b. As $\theta^{\prime}=\theta+\delta=0, \alpha_{1}=\cos \theta^{\prime}=1$, and $\alpha_{2}=\sin \theta^{\prime}=0$.

It needs to stress again the significant result just obtained that a dynamical state of the $\mathrm{M}$ phase represented by any eigenket of perturbed $\overline{\overline{H_{1}}}$ is the superposition of the two eigenkets of $\overline{\overline{\mathcal{H}}}$ in the unperturbed state. The salient fact is that the $\mathrm{M}$ ensemble is accordingly a new phase and its structure is essentially different from those of 1 and 2 phases but explicitly related to them. This is the unique evidence of the dynamical state of the $\mathrm{M}$ phase verified by the quantum mechanical idea; showing good contrast with that from thermodynamical one. According to the latter, the pure 1 and 2 phases must coexist independently in the $\mathrm{M}$ phase, and do not produce a new phase having its unique structure.

The thermal change of the distribution coefficients $\alpha_{1}$ and $\alpha_{2}$ in a temperature interval $\Delta T$ of the $\mathrm{M}$ phase are indicated in Figure 9. It is very important that the crystal of the $\mathrm{M}$ phase exhibits perceptibly changing structure in accordance with the temperature dependence of $\alpha_{1}$ and $\alpha_{2}$.

\subsection{Temperature variation of the specific heat}

For assessing the validity of the present theory, we will examine first whether or not it explains the extraordinary thermal dependence of specific heat $C_{p}$ that has already 


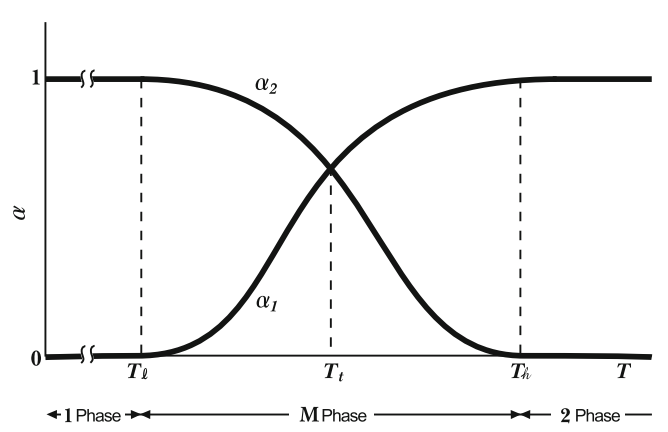

Fig. 9. Temperature variation of relative weight $\alpha_{1}$ and $\alpha_{2}$ of eigenfunctions of Hamiltonian of 1 and 2 ensembles.

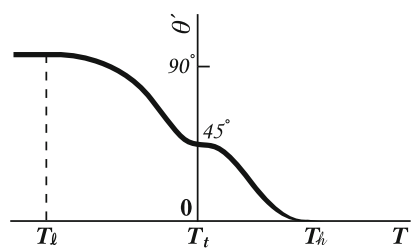

(a)

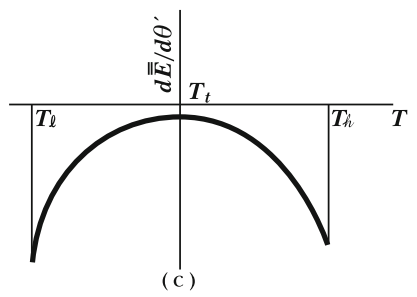

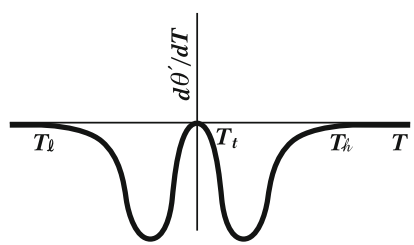

(b)

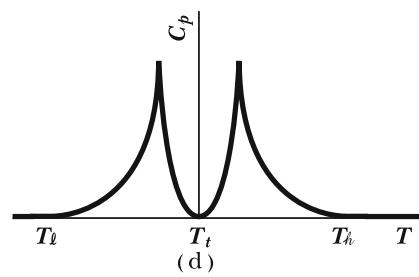

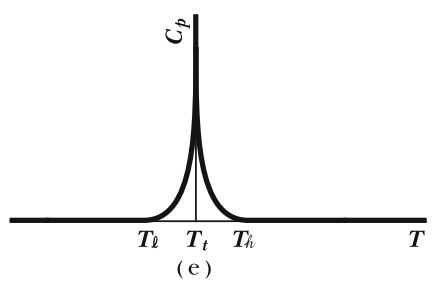

Fig. 10. Derivation of temperature change of specific heat $C_{p}$. $\theta^{\prime}$ vs. $T$, (b) $d \theta^{\prime} / d T$ vs. $T$, (c) $d E / d \theta^{\prime}$ vs. $T$, (d) $C_{p}$ vs. $T$, (e) $C_{p}$ vs. $T$ for $\Delta T \sim 0$.

been mentioned. For brevity, let the mechanical work done by the crystals be unconsidered. Then $C_{p}$ is expressed as

$$
C_{p}=\frac{d \overline{\bar{E}}}{d T}=\frac{d \overline{\bar{E}}}{d \theta^{\prime}} \frac{d \theta^{\prime}}{d T}
$$

where $\theta^{\prime}=\cos ^{-1} \alpha_{1}=\sin ^{-1} \alpha_{2}$.

The thermal dependence of $\theta^{\prime}$ can be estimated qualitatively from the processes of constructing Mohr circles. The result is shown in Figure 10a. $\theta^{\prime}$ which is $90^{\circ}$ at $T_{l}$ (Fig. 5), decreases as $T$ increases, levels off $45^{\circ}$ at $T_{t}$ as can be readily recognized from Figure $4 \mathrm{a}$, finally vanishes at $T_{h}$. From this figure $d \theta^{\prime} / d T$ can be evaluated as depicted in Figure 10b. It is noticeable that the two negative peaks are formed between $T_{l}$ and $T_{t}$ and between $T_{t}$ and $T_{h}$, nearly symmetrical with respect to the ordinate at $T_{t}$.

The thermal variation of $d \bar{E} / d \theta^{\prime}$ in the range of $\Delta T$ of the $\mathrm{M}$ phase can be evaluated in the following.

(i) $T_{l}<T<T_{t}$.

The thermal change of internal energy $\Delta \overline{\bar{E}}$ of the M phase is expressed as

$$
\Delta \overline{\bar{E}}=\alpha_{1} \overline{\overline{E_{1}}}-\left(1-\alpha_{2}\right) \overline{\overline{E_{2}}}=\overline{\overline{E_{1}}} \cos \theta^{\prime}+\overline{\overline{E_{2}}} \sin \theta^{\prime}-\overline{\overline{E_{2}}} .
$$

Hence

$$
\frac{d \overline{\bar{E}}}{d \theta^{\prime}}=-\overline{\overline{E_{1}}} \sin \theta^{\prime}+\overline{\overline{E_{2}}} \cos \theta^{\prime}
$$

which manifests a negative peak of $-\overline{\overline{E_{1}}}$ at $T_{l}$ and becomes $1 / 2\left(\overline{\overline{E_{2}}}-\overline{\overline{E_{1}}}\right)$ at $T_{t}$.

(ii) $T_{t}<T<T_{h}$

$$
\begin{aligned}
\Delta \overline{\bar{E}} & =\left(1-\alpha_{1}\right) \overline{\overline{E_{1}}}-\alpha_{2} \overline{\overline{E_{2}}} \\
& =\overline{\overline{E_{1}}}-\left(\overline{\overline{E_{1}}} \cos \theta^{\prime}+\overline{\overline{E_{2}}} \sin \theta^{\prime}\right) .
\end{aligned}
$$

Hence

$$
\frac{d \overline{\bar{E}}}{d \theta^{\prime}}=\overline{\overline{E_{1}}} \sin \theta^{\prime}-\overline{\overline{E_{2}}} \cos \theta^{\prime},
$$

which becomes a negative peak of $-\overline{\overline{E_{2}}}$ at $T_{h}$. The temperature dependence of $d \overline{\bar{E}} / d \theta^{\prime}$ is depicted in Figure 10c. Finally the temperature dependence of $C_{p}$ is obtained by making the product of $d \theta^{\prime} / d T$ and $d \overline{\bar{E}} / d \theta^{\prime}$ at each temperature. The appearance is shown in Figure $10 \mathrm{~d} . C_{p}$ shows clearly two peaks nearly symmetrical with respect to the ordinate at $T_{t}$. As $\Delta T$ is shortened, the two peaks approach together and finally overlap to appear a nearly single peak as shown in Figure 10e. Thus the present theory resolves the reason why the specific heat manifests sometimes two split peaks, while sometimes apparently a single peak at $T_{t}$.

\subsection{Temperature dependence of extensive physical properties}

Consider an extensive physical property of $A$ as in chapter 3.1. The macroscopically observed value of $A$ in the M phase is an ensemble averaging $\overline{\bar{A}}$, which is expressed in (10). Therefore $\overline{\overline{A_{M}}}$ at any temperature in the M phase is expressed as

$$
\overline{\overline{A_{M}}}=\operatorname{Tr} \rho_{M} A
$$

where $\rho_{M}$ is the density matrix of the M phase.

The figure of $\overline{\overline{A_{M}}}$ cannot be inferred from $\overline{\bar{A}}$ of the 1 and 2 phases except cases of approaching closely these phases, because the structure of the M phase differs from 

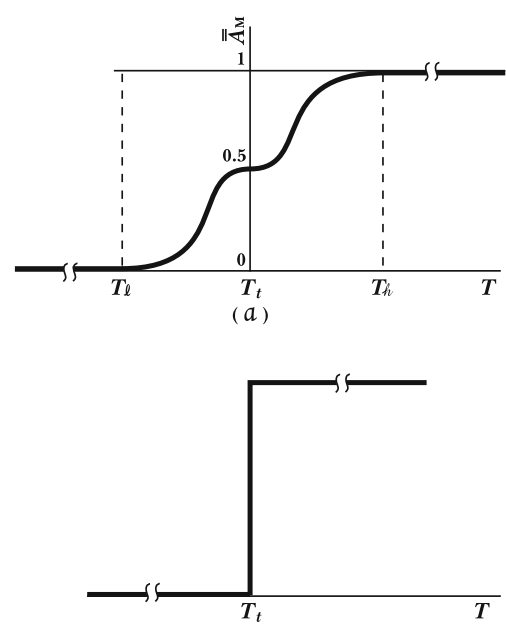

(b)

Fig. 11. Temperature dependence of an external physical property $A_{M}$ in M phase.

either 1 and 2 phases. The characteristic feature of $\overline{\overline{A_{M}}}$ is that it consists of the two parts separated at $T_{t}$. They are related by inversion symmetry, because values of $V$ of the 1 and 2 phases are nearly related by reflection symmetry at $T_{t}$, and $\alpha_{1} / \alpha_{2}$ and $\alpha_{2} / \alpha_{1}$ of both parts are also operated by the same symmetry. An arbitrary example of $\overline{\overline{A_{M}}}$ is drawn in Figure 11a. In cases that $\Delta T$ is small, $\overline{\overline{A_{M}}}$ would appear to change abruptly at $T_{t}$ as shown in Figure $11 \mathrm{~b}$.

Summing-up, the present theory explains most of aberrant experimental phenomena occurring at FOPT, on which long issues have been expended as described in Chapter 2.

\section{Typical examples with discernible $M$ phase}

\subsection{Dichlorobenzophenone (DBP)}

Dichlorobenzophenone manifests very interesting FOPT phenomena. X-ray studies were made mainly by Mitkevich et al. [7] and Zuninga et al. [8]. The crystal shows definite hysteresis of transitions at about $186 \mathrm{~K}$ (cooling) and $192 \mathrm{~K}$ (heating). The space groups of 1 and 2 phases are $I 2 / c$ and $C 2 / c$ respectively. The temperature variations of lattice constants are shown in Figure 12. It is conspicuous that those of $a$ and $\beta$ are closely similar to Figurer 11a, but others seem experimentally inaccurate.

An important evidence of the FOPT of this crystal was revealed from NQR spectroscopy. Although single $\mathrm{Cl}$ line spectrum in each 1 and 2 phase is observed, four spectra were measured in the intermediate phase [9]. It is known that the NQR spectrum outlines the microscopic environment of the resonant nuclei. In a commensurate system the number of NQR lines is determined by the nonequivalent nuclear sites in the unit cells.

Mitkevich et al. [7] reported that the intermediate state must be a mixture of the quasi- $C$ and quasi- $I$ lattices. Zuninga et al. [8] showed that it was monoclinic
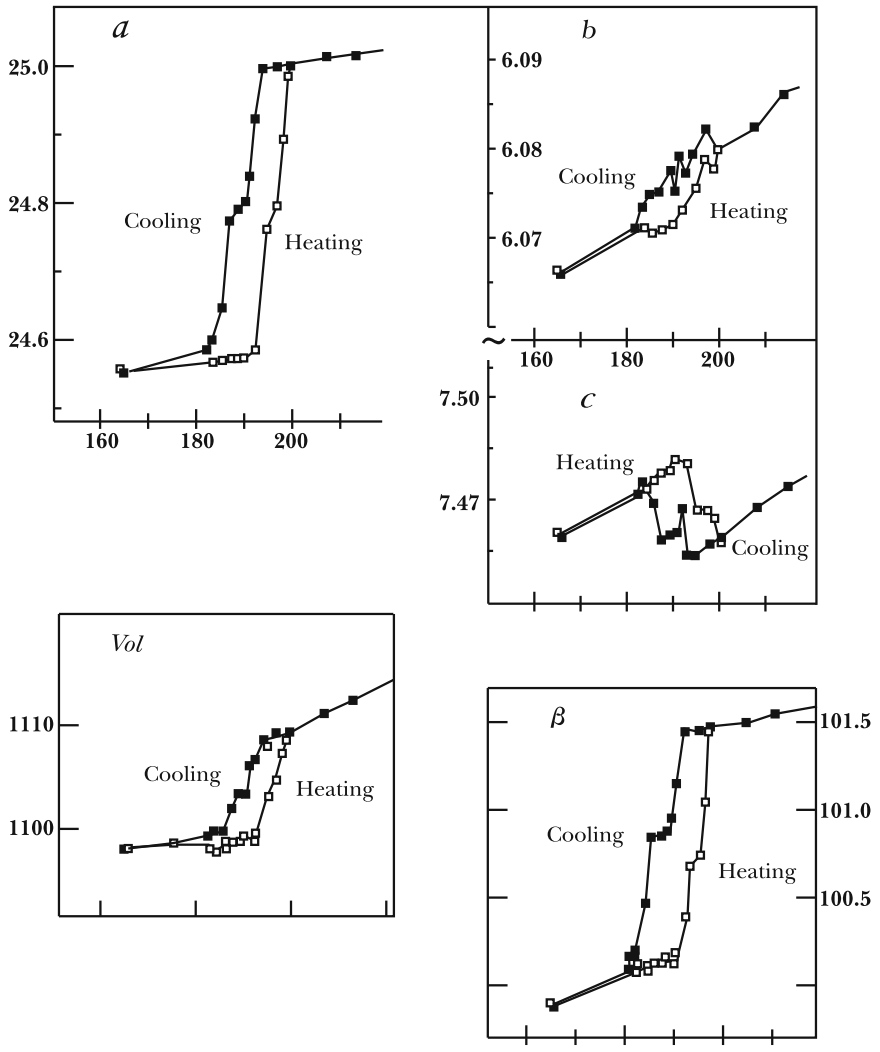

Fig. 12. Temperature dependence of lattice parameters of dichlorobenzophenone (after Herbstein [2]).

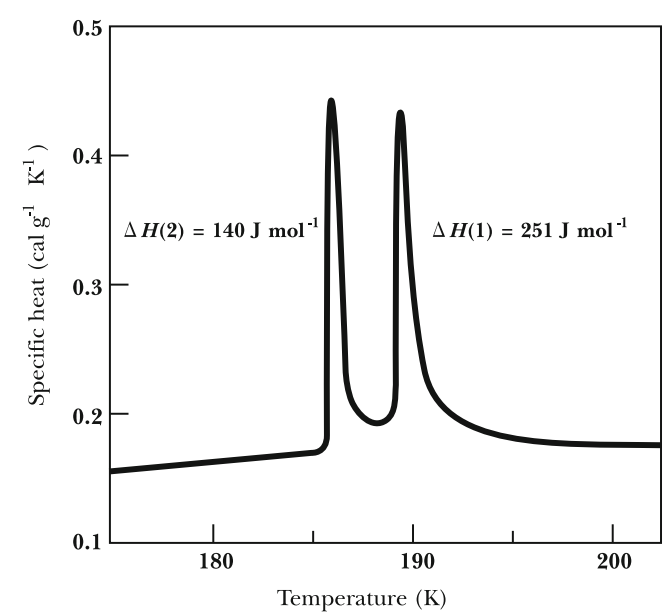

Fig. 13. Temperature dependence of specific heat of dichlorobenzophenone (after Herbstein [2]).

but primitive and that new superlattice reflections appear. They insisted that this structure is still a sort of coexistence of domains of $I$ - and and $C$-centering zones of alternated cells CICI... As a conclusion, one cannot describe the intermediate state beyond noting that there seem to be four independent $\mathrm{Cl}$ atoms and that the structure appears to be modulated.

The temperature variation of specific heat was measured by Ecolivet et al. [10] as shown in Figure 13, where 

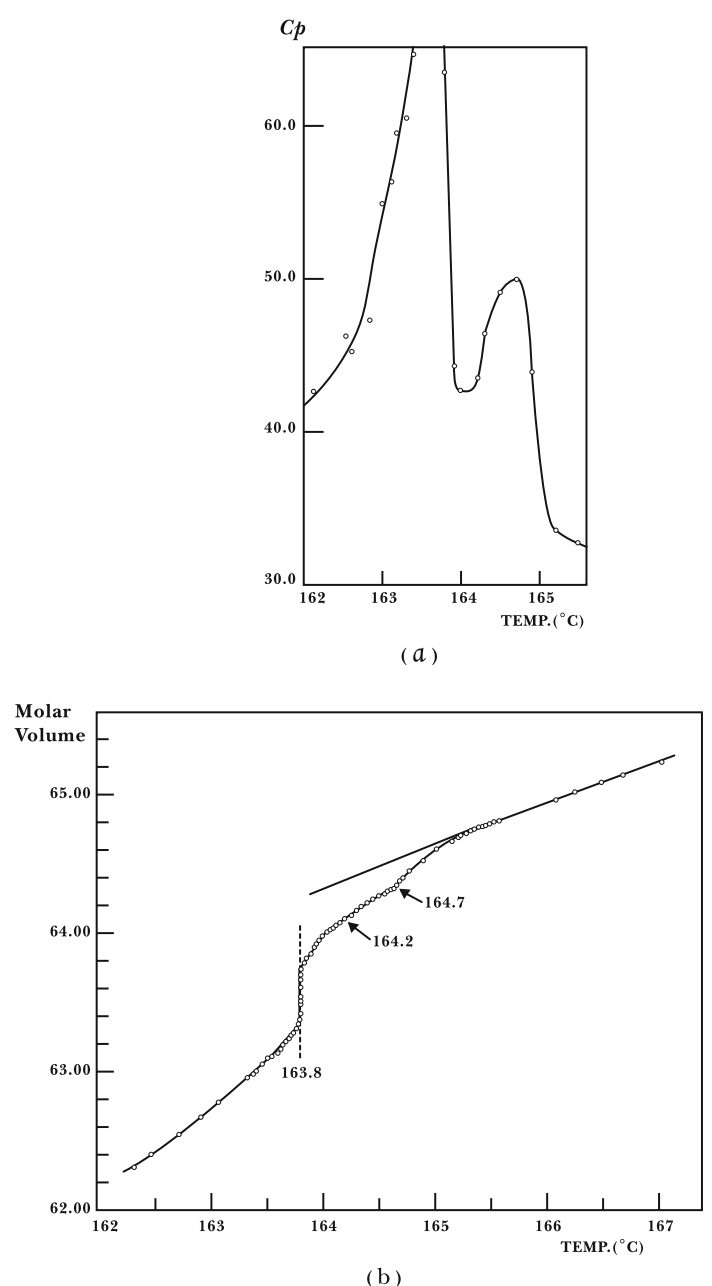

Fig. 14. Calorimetric and dilatometric behavior of $\mathrm{NaNO}_{2}$. (a) specific heat vs. $T$, (b) volume vs. $T$ (after Sakiyama [11]).

splitting of peaks is clearly seen. The overall situation is described by Mitkevich [7]: the phase transition occurs as a sequence of two separate FOPTs with an intermediate state between 1 and 2 phases. We should like to point out that the intermediate phase of DBP is undoubtedly the M phase and its behaviors agree considerably well with our prediction from the quantum theory.

\section{$4.2 \mathrm{NaNO}_{2}$}

$\mathrm{NaNO}_{2}$ was found to undergo FOPT around $163{ }^{\circ} \mathrm{C}$ by Sawada et al. [11]; the low temperature Im $2 m$ phase is ferroelectric, and the high temperature $I m m m$ phase is paraelectric. Tanisaki [12] reported the presence of a new intermediate phase in the temperature region of $163-164{ }^{\circ} \mathrm{C}$ from X-ray studies. Sakiyama et al. [13] made calorimetric and powder dilatometic studies. They found splitting of heat capacity around $163{ }^{\circ} \mathrm{C}$ as shown in Figure 14a, and unusual volume vs. temperature curve in Figure 14b. It seems that the behavior of volume is approximately similar to Figure $11 \mathrm{a}$ except abrupt change at $163{ }^{\circ} \mathrm{C}$. It can be said that the presence of double peaks of $C_{p}$ and of

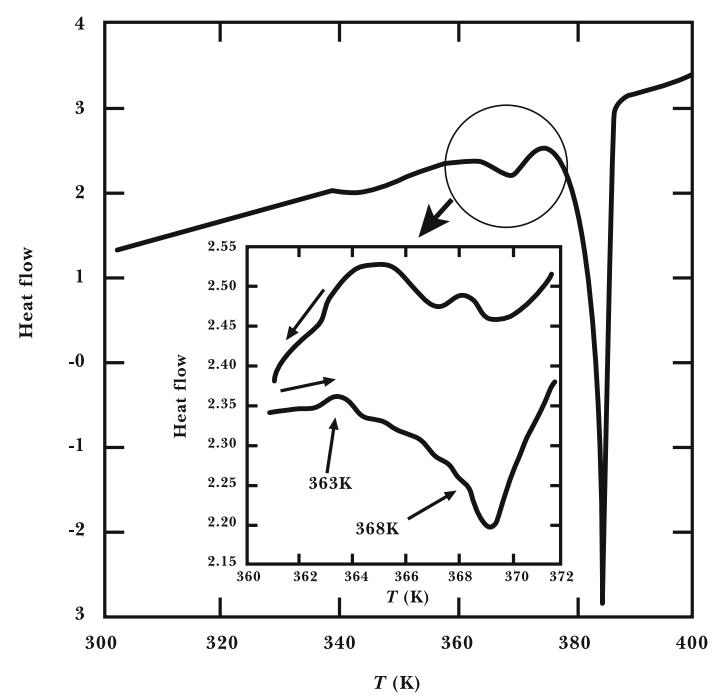

Fig. 15. DSC curve of 1-Ethyl-3-(4-methylpentanoyl)(after Herbstein [2]).

apparent thermal tendency of volume support the generation of $\mathrm{M}$ phase. Besides, Yamada et al. [14] propsed that the $\mathrm{M}$ phase has unique structure, antiferroelectric with a sinusoidal modulation of $\mathrm{NO}_{2}$ ions.

\subsection{1-Ethyl-3-(4-methylpentanoyl)urea}

Crystal melting is the most magnificent FOPT, accordingly attracts great interest. Hashizume et al. [15] carefully studied FOPT of 1-Ethyl-3-(4-methylpentanoyl)urea by calorimetric and X-ray methods. Their DSC curve of this crystal is shown in Figure 15. There are two splitted peaks at around $369 \mathrm{~K}$ and $384 \mathrm{~K}$ (melting). We can immediately recognize that these peaks represent the characteristic splitting of $C_{p}$ in the $\mathrm{M}$ phase. Hashizume et al. analyzed thermal structure change by precisely temperatureresolved diffraction method at every $2 \mathrm{~K}$ in the range $348-374 \mathrm{~K}$.

Hashizume et al. [15] found that the crystal is isostructural with space group of $\boldsymbol{P} 1$ before and after $363 \mathrm{~K}$, but abrupt change of lattice constants occur from type I unit cells to type II unit cells. Structural change is remarkable; molecular structure of the I region changes into supramolecular structure of II. The change of the intermolecular geometries defined by $(\mathrm{I})-(\mathrm{V})$ say is shown in Figure 16. It is especially worth-noting that the supramolecular structure (M phase) makes rapid thermal change as expected by the theory. This experiment may provide the first proof of characteristic change of $\mathrm{M}$ phase structure.

\section{$4.4 \mathrm{VO}_{2}$}

When an insulator-to-metal transition is induced in correlated insulators or Mott insulators by doping or heating, the resulting conducting state is known radically different 


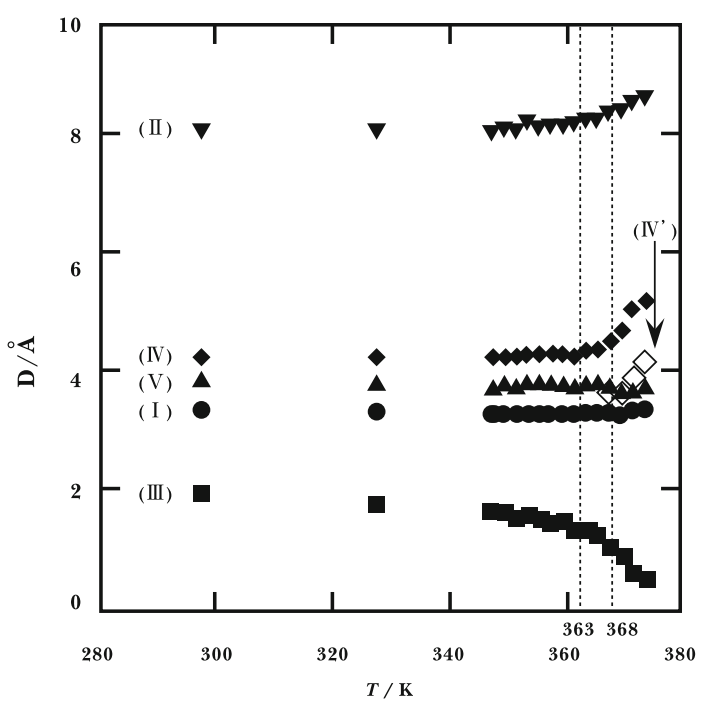

Fig. 16. Temperature dependence of the various intermolecular contacts and packing geometries of 1-Ethyl-3-(4methylpentanoyl)urea (after Hashizume [15]).

from that characterized by free electrons in conventional metals. Qazilbash et al. [16] studied prototypical correlated insulator $\mathrm{VO}_{2}$ in which the metallic state can be induced by only increasing temperature.

Using scanning near-field infrared microscope, they found nucleation of nanoscale metallic puddles in the host of the low temperature monoclinic phase slightly below the transition temperature of $T_{t} \sim 342 \mathrm{~K}$. Puddles grow and connect with themselves within narrow temperature region of $\Delta T \sim 2 \mathrm{~K}$. The insulator-to-metal transition is followed by severely sudden fading and percolative approach to $360 \mathrm{~K}$, whence the crystal becomes perfectly rutile type conducting phase. Qazilbash et al. [16] also performed detailed far-infrared spectroscopy on the same specimen over all the transition temperature range. The nature of nanostructural puddles regions were separated theoretically from that of the host phase by use of an effective medium theory $[17,18]$. It is,of course, a natural prediction that the effective optical constants of these heterogeneous system are an average of the optical constants of the insulating and rutile metallic regions weighted by respective volume fractions. However, they reached very important conclusions. This simple evaluation of optical constants of the insulating phase and of the rutile metallic phase at $360 \mathrm{~K}$ does not produce a satisfactory description of the far-field infrared data near the onset of insulator-tometal transition of $\mathrm{VO}_{2}$. This discrepancy indicates that the infrared properties of the nanoscaled metallic puddles, once they appear at $342 \mathrm{~K}$, may be different from those of the high-temperature rutile metal.

The resistance-temperature curve showing the insulator-to-metal transition is indicated in Figure 17. The monoclinic insulator to rutile metal transition is unambiguously interleaved by the strongly correlated metal within $\Delta T \sim 2.2 \mathrm{~K}$. Qazilbash et al. point out that the behavior of insulator-to-metal transition of $\mathrm{VO}_{2}$ displays clearly Mott transition feature.

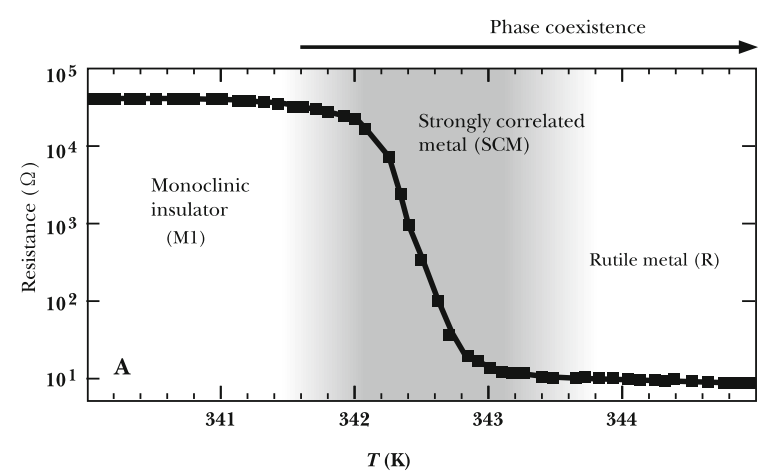

Fig. 17. The phase diagram of $\mathrm{VO}_{2}$ and the resistance vs. temperature curve showing the insulator-to-metal transition (after Qazilbash et al. [16]).

It seems likely to us that insulator-to-metal transition corresponds to the formation of the $\mathrm{M}$ phase, since the structure of transforming phase is entirely different from those of 1 and 2 phases, and the shape of temperature variation of resistance (Fig. 17) resembles Figure 11a. The percolative regime over relatively long temperature range cannot be precisely understood but appear to be subsidiary effect. Then it may be inferred that the Mott transition of $\mathrm{VO}_{2}$ attributes to the $\mathrm{M}$ phase formation.

Baum et al. [19] reported the temperature change of atomic positions of $\mathrm{VO}_{2}$ by using femtosecond electron diffraction. This specimen at room temperature was irradiated near-infrared pulses whose one pulse energy is enough to drive a phase transition. They were successful in delineating consecutive atomic shifts within $1 \mathrm{ps}$. The primary shift is relaxing $\mathrm{V}-\mathrm{V}$ pair bonds along the $a$ axis in the low temperature phase without accompanying substantial lattice expansion. Another shift of $\mathrm{V}$ atoms takes place along the $c$ axis direction with slow sound wave velocity of 9.2 ns of time constant, thus completing the transition to the rutile structure. This shift is accompanied by shear motion of the lattice.

It is of specific interest that the direction of the first antibonding shift is rectangular to shear forming shift. Therefore it may be that the antibonding motion is driven by perturbing energy and fulfills primary prerequisite for the Mott transition. This temporal experiments of FOPT is different object from the present study but very useful for getting dynamical knowledges of FOPT on atomic levels.

\section{Discussion}

The present theory reaches a new interpretation on apparently aberrant phenomena occurring at FOPTs of crystals. At a FOPT an intermediate $\mathrm{M}$ phase is generated as a dynamic state of superposing eigenfunction of average Hamiltonian eigenfunctions of low and high temperature phases. The primary requisite for the $\mathrm{M}$ phase formation is a weak interaction between two eigenstates of $\overline{\overline{H_{1}}}$ and $\overline{\overline{H_{2}}}$. However, we feel it necessary to notice that such a kind of effect is not specifically limited in FOPT in crystals. 
A similar effect gives rise to other significant phenomena. For instance, such interactions always exist between eigenstates of optical impermeability in a crystal, thus producing optical activity in the crystals. However, optical activity can be found only in 15 noncentrosymmetric classes due to nullification of the effect by the symmetry operations in other classes. The emergency of incommensurate state of some ferroelectric crystals indicates an additional example. We [20] found that its origin is lifting of a specific lattice vibrational mode degenerating with its conjugate star as a result of time-reversal symmetry.

Correct clarification of the atomistic mechanisms of generating $M$ phases is highly desirable since it provides insight into origins of phase transition mechanisms that have remained uninvestigated. The crystal structure in the $\mathrm{M}$ phase, as partly described in chapter 4, certainly demonstrates the difference from a simple coexistence of 1 and 2 phases even in previous investigations; for example, structural modulation and supramolecular structure formation of 1-Ethyl-3-(4-methylpentanoyl)urea may be mostly decisive.

The recent experiments on $\mathrm{VO}_{2}$ by Qazilbash et al. [16] reveal an especially significant aspect of the $\mathrm{M}$ phase in a correlated insulator. It emerges and proceeds through the process of nucleation-and-growth. However, the growing domains are not high temperature phase but the nanoscale $\mathrm{M}$ phase. Therefore the mechanism appears different from the nucleation-and-growth model proposed by Mnyukh [4]. It is a very interesting that domains of $\mathrm{VO}_{2}$ appear in the host phase but their structure differs from that of high temperature form.

The author expresses his sincere gratitude to Prof. S.C. Abrahams, who provided him with the impetus of beginning the present work.

\section{References}

1. P. Ehrenfest, Proc. Acad. Sci. Amsterdam 36, 153 (1933)

2. F.H. Herbstein, Acta Cryst. B 62, 341 (2006)

3. A.R. Ubbelohde, Nature (London) 169, 832 (1952)

4. Yu.V. Mnyukh, N.A. Panfilova, J. Phys. Chem. Solids 34, 159 (1973)

5. C.N.R. Rao, K.J. Rao, Phase Transitions in Soilds (Mc Graw-Hill Inc., 1978)

6. R.J. Gooding, J.R. Morris, Phys. Rev. E. 47, 2934 (1993)

7. V.V. Mitkevich, V.G. Lirtsman, M.A. Strzhemechny, A.A. Avedeeko, V.V. Ermenko, Acta Cryst. B 55, 799 (1999)

8. F.G. Zuninga, A. Criado, Acta Cryst. B 51, 880 (1995)

9. A.E. Wolfenson, D.J. Pusiol, A.H. Brunett, Naturforsch. Teil. A 45, 334 (1990)

10. C. Ecolivet, M. Bertaut, A. Mierzejewski, Dynamics of Molecular Crystals (Elsivier, Amsterdam 187, 1987)

11. S. Sawada, S. Nomura, S. Fujii, I. Yoshida, Phys. Rev. Lett. 1, 320 (1958)

12. S. Tanisaki, J. Phys. Soc. Jpn 16, 579 (1961)

13. M. Sakiyama, A. Kimoto, S. Seki, J. Phys. Soc. Jpn 20, 2180 (1965)

14. Y. Yamada, I. Shibuya, S. Hoshino, J. Phys. Soc. Jpn 18, $1594(1963)$

15. D. Hashizume, N. Miki, T. Yamazaki, Y. Aoyagi, T. Arisato, H. Uchiyama, T. Endo, M. Yasui, F. Iwasaki, Acta Cryst. B 59, 404 (2003)

16. M.M. Qazilbash, M. Brehm, Byung-Gyu, P.C. Ho, G.O. Andreev, Bong-Jun Kim, SunJin, Yun, A.V. Balatsky, M.B. Maple, F. Keilmann, H.T. Kim, D.N. Bosov, Science 318, 1750 (2007)

17. See Materials and Methods on Science Online

18. G.L. Carr, S. Perkowitz, D.B. Tnner, Infrared and Milimeter Waves (Academic Press, Orlando, 1985), Vol. 85

19. P. Baum, D.S. Yong, A.H. Zewail, Science 318, 788 (2007)

20. J. Kobayashi, Phys. Rev. B 42, 8332 (1990) 